\title{
Design of an economical and reliable net-metering device for residential consumption measurement using IoT
}

\author{
Hassan Jamal ${ }^{1}{ }^{*}$, M. Nauman Arshad ${ }^{2}$,Yasir Butt ${ }^{3}$, Hasan Shafiq ${ }^{4}$, Abdul Manan ${ }^{3}$, Akash Arif ${ }^{3}$, Mohsin Khan Janjua ${ }^{5}$ \\ Technical University of Munich (Power Engineering) ${ }^{1}$, Bilkent Univerisity (Mechanical Engineering) ${ }^{2}$, UET, Taxila (Electrical \\ Engineering) ${ }^{3}$, University of Lahore (Aviation) ${ }^{4}$, NUST, H-12 (Energy Engineering) ${ }^{5}$ \\ hassan.jamal@tum.de, hassan.jamal@ieee.org, $\underline{\text { ORCID }}$ *
}

\begin{abstract}
With the proliferation of decentralised power generation technologies and smart grid infrastructures, residential renewable energy generation (REG) has burgeoned to become intrinsic to modern power grids and is expected to play a fundamental role for the realization of netzero energy buildings to cater to the ever-escalating energy demand. The integration of domestic REG into the utility grid necessitates a medium of intelligent devices to perform net metering operations. Henceforth, we propose one such cost effective smart net-metering device for household REG systems with in-built wireless bi-directional communication based on Internet of Things (IoT) technology. Net metering operations are implemented using a microcontroller to synchronously collect, analyze and transmit data from sensors installed at the utility to consumer and REG to utility sides to both an offline display and an online server via a WiFi module interfaced to the microcontroller. We successfully implemented a prototype device for real-time energy monitoring over a sustained period of time, yielding accurate power generation and energy transfer statistics with remotecontrol operations. These results demonstrate the considerable potential for the prototype to be a stimulant in catalysing our eventual shift to decentralised power generation.
\end{abstract}

Keywords - net metering, internet of things, net-zero energy, energy tariffs, microcontroller

\section{INTRODUCTION (HEADING 1)}

Due to ubiquitous environmental, socio-ethical and economical sustainability concerns, conventional dominant non-renewable centralized energy generation systems are being rapidly replaced by distributed renewable energy (DRE) systems [1]. Whereas, decentralized generation of power is also intrinsic to efforts aimed at minimizing power generation from fossil fuels, it necessitates high penetration of REG from urban domestic households to utility grids, wherein they act as highly-dense local power generators to ultimately realize net-zero energy urban regions $[1,2]$. A recurring challenge in facilitating this bi-directional power flow between the consumer and utility is a modernization of net-metering techniques that essentially govern the quintessential stimuli for REG, such as rebate programs and net-zero energy buildings [3].

Pakistan possesses huge potential for REG owing to its suitability for solar power generation and the presence of dense urban centers [4]. However, the widespread propagation of REG is hindered significantly by traditional meter reading practices that are both time-consuming and require the deployment of extensive human resources. Then there is the all-pervading issue of accuracy due to prevalent corrupt practices and potentially exacting loads on a dwindling human resource whereby only a few personnel are deployed for a large area. The outcome is general misreporting of energy consumption leading to erroneous statistics for future planning and developments in the power sector. Concurrently, this also leaves potential back-doors for energy theft and other malpractices $[5,6]$.

Achieving a high penetration of DER at the low-voltage network stands upon incentivizing it for the urban producer and advanced metering infrastructure. A smart net metering system would not only provide the gateway for the domestic REG to export energy to the grid with efficient net metering operations but also enable the remote monitoring of net-metering parameters [6]. Pacis et al, worked on the integration of Zigbee technology with conventional net meters for the wireless monitoring and reading of surplus energy generated through photovoltaic systems [7]. Similarly, Rahman had proposed the design of net meters using Global System for Mobile Communication (GSM) technology, which transmits wireless information about energy exchange between the domestic REG and utility in kWh [8]. Moreover, Desker et al and Maharaja et al highlighted the design of a bidirectional net-metering system using FPGA for a microgrid. The designed meter computes the transmission of energy between microgrids with multiple users and utility grids using wireless protocols $[9,10]$.

However, there is yet to be a system that wirelessly monitors and transmits the net meter readings to both the utility and producer. Furthermore, Ostia et al have discussed the development of a smart controller integrated with a hybrid net metering system. Their work primarily focuses on assistance in the controlling of battery charging from solar energy and monitoring the battery state of charge, voltage and energy consumption in $\mathrm{kWh}$ on the designated web graphical user interface (GUI) [11].

Thus, an overview of recent literature reveals that the majority of the research work focused on the development of the net metering system, whereas few researchers focused on developing smart systems for producers and utility operators to perform real-time analysis of their energy transmission. Additionally, current net metering systems are highly expensive and involve low range wireless devices and protocols such as Zigbee, Xbee and Bluetooth including some outdated and high-power consuming wireless modules (e.g. GSM) for the global monitoring of net meters using text messaging [7, 8, 9 and 10]. Moreover, IoT technology has not been yet integrated into net metering systems for the remote-control operations and virtual monitoring of multiple metering parameters.

This study mainly focuses on the amalgamation of IoT technology with a reliable and cost-effective net energy metering device for domestic REG. The integration of IoT technology will allow the designated net meter to communicate globally via Blynk protocols with both the utility control center and the REG owners. The smart net 
meter sends real-time data related to total renewable generation, load demand, surplus renewable energy shared with the utility, utility energy consumption and as well as the total cost of energy shared or received with or by the utility. Moreover, it allows REG owners or utility operators to perform remote operations to terminate the supply of REG to the utility grid in case of technical faults or maintenance. Furthermore, the designed system permits the REG owners to perform real-time offline monitoring of the aforementioned net metering parameters and control operations using a high-resolution tactile liquid crystal display. The sustainability, sensitivity, accuracy and robustness of the developed system was continuously tested for a duration of 6 hours under multiple loads, REG and utility energy trade configurations to simulate real-life operation.

\section{SYSTEM DESCRIPTION}

The proposed IoT-based net metering system can be divided into four main parts as illustrated in the figure: the utility grid, the smart net meter and the residential load, DC to AC Inverter and PV array unit.

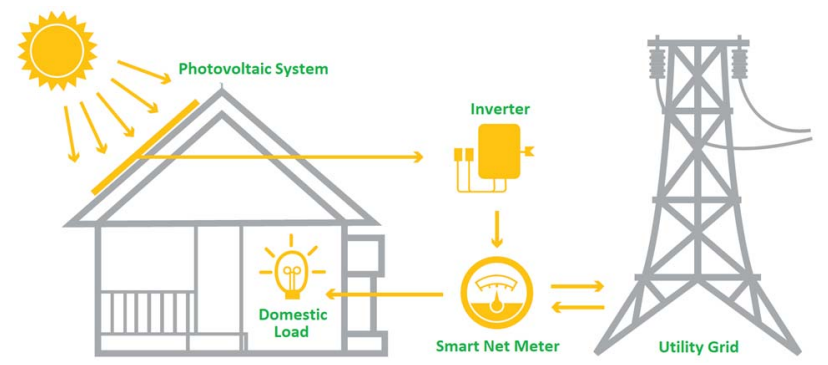

Fig 1. Visual representation of a designed net metering system

\section{A. Utility Grid}

Since the experimentation was performed exclusively within Pakistan, the corresponding parameters are used throughout the paper. In Pakistan, the utility grid is a 3phase supply system with a nominal voltage of $220 \mathrm{AC}$ line-to-ground and $440 \mathrm{AC}$ line-to-line. Most domestic to grid power connections in Pakistan are, however, singlephase connections and thus, require a single-phase energy meter for power usage computations [12]. Thereby, this study is focused on the designing and testing of a singlephase net-metering system but it can be easily modified for use with the multifarious utility grid configurations used around the world.

\section{B. Smart Net Meter}

The smart net meter in this study represents the control units, sensors and wireless communication modules for complex power metering computations and operations. These computations are carried out with the Node MCU (ESP-12E) [13] as the net meter in conjunction with the Blynk server and the Blynk mobile application to incorporate the wireless transmission of data from and to the utility grid, domestic REG and the subsequent revision of parameters, if needed [14].

\section{Domestic Loading \& PV System}

The nature of domestic loading can be simplified to a combination of inductive and resistive loads. Consequently, even though the influence of inductive loading has adverse effects on the power factor $(\mathrm{PF})$ of the entire individual domestic load, this is considerably lower compared to its effects on commercial loading. Therefore, the domestic tariffs are calculated such that the effects of reactive power or PF on the overall behaviour of apparent power consumed are discarded. As a result, monthly energy billing for the residential sector assumes domestic loading as a resistive loading entirely $[15,16]$. Hence, to maintain consistency with local practices, only the effect of resistive loading was considered in all the experiments for the proposed smart net-metering system. Moreover, for the sake of lucidity, this study assumes pure sine wave DC to AC inverters for energy conversion from monocrystalline photovoltaic cells.

\section{Technical Overview}

\section{a. Internet of Things}

The Internet of Things (IoT) is an evolution of conventional technologies such as ubiquitous computing, Machine-to-Machine (M2M), and embedded Internet systems, which enables the multi-directional global communication between various physical devices (which contains controlling and sensing units) over the internet. In smart grids, the IoT technology is being increasingly utilised to monitor parameters of various electrical devices and to perform remote-control operations in order to enhance the sustainability and efficiency of the entire power network. This study uses wireless communication technology in collaboration with Blynk HTTP protocols to enable IoT operations.

b. Blynk

Blynk is an IoT platform that supports both iOS and Android while being compatible with a plethora of microcontrollers such as Node MCU (ESP), STM32, Arduino and Raspberry $\mathrm{Pi}$ over the Internet. The architecture of Blynk consists of three major components: 1) the Blynk application, which controls an embedded system and displays sensing data on widgets; 2) the Blynk server, which allows all cloud-based communications between smartphones and embedded systems; and 3) the Blynk libraries, which consist of various widgets to perform different control, display, and time management operations. The details are illustrated in figure 2 .

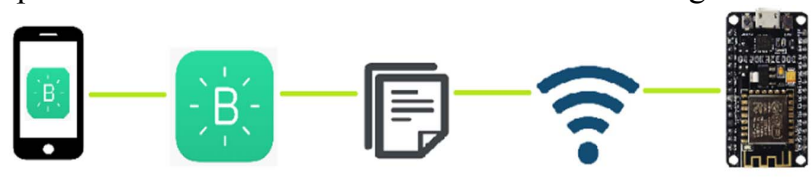

Blynk App Blynk Server Blynk Libraries Internet Access IoT Module Fig 2. Blynk working principle

\section{c. Node MCU ESP $12 E$}

To ensure a robust, affordable, smart and stable net metering system, the Node MCU (ESP-12E) microcontroller was chosen owing to its many advantages over other microcontrollers such as its built-in IEEE802.11b/g/n 2.4 GHz Wi-Fi module that supports $+19.5 \mathrm{dBm}$ output at the antenna, its $160 \mathrm{MHz}$ single-core CPU with $4 \mathrm{MB}$ of flash memory, intrinsic to the stable operation of a smart net metering system due to the presence of an assortment of complex computations, and 
its wide operating temperature range of -40 to $125^{\circ} \mathrm{C}$, essential for robustness $[13,17]$.

\section{d. Sensors}

Off the non-exhaustive list of major sensors used and proposed in this research for calculation of power transmission at domestic REG and utility side, two are worth special mention: the ACS712 AC current sensor and ZMPT101B AC voltage sensor. The ACS712 sensor measures AC currents of up to 30 amps with a scale factor of $66 \mathrm{mV}$ per amps [18], whereas, the ZMPT101B sensor has the capability to measure AC voltages of up to 250 volts with $0.02 \mathrm{~V} \mathrm{DC} / 1 \mathrm{~V}$ AC scale factor [19]. Both sensors are highly precise over experimental temperatures $[18,19]$.

\section{DESIGN \& WORKING PRINCIPLE}

To enable real-time remote control operations, monitoring of power exchange between residential REG and the utility grid and to perform net metering operations, a control unit was designed such that the REG owner had fully autonomous access whereas the utility grid operator had partial access. The control unit of the net metering system includes a logically programmed Node MCU (ESP $12 \mathrm{E})$ to perform essential operations for a sustainable energy transfer: synchronized sensor monitoring, smart actuation, wireless data operations, ancillary graphic display and miscellaneous net metering computations. Figure 3 demonstrates an abridged workflow of the entire net metering control unit.

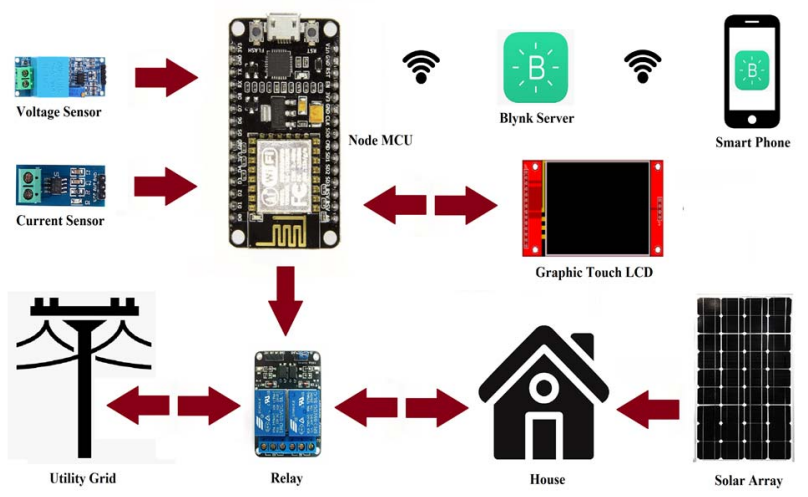

Fig 3. Working flow of designed net metering control unit

Current and voltage transducers deployed at the REG and utility grid sites synchronously transmit voltage and current values to the Node MCU microcontroller wherein these values are used to compute the phase angle, voltage waveform, total REG, instantaneous load demands, total energy exported/imported to/from of the utility grid and simultaneously provide the net cost of energy shared or received from the utility.

The calculated values are then disseminated wirelessly to the Blynk server through the Blynk HTTP protocols and Blynk libraries. The uploaded data at Blynk server is then metamorphosed to the high-resolution graphic widgets on the Blynk app and touch LCD for offline local data monitoring and real-time operations. In case of technical faults or maintenance, the Blynk remote-control switch allows REG user to abort the connection between utility grid and domestic REG using electromechanical relays.

\section{A. Net-Metering Mathematical Model}

A synopsis of the mathematical model developed for the supplementary energy from REG which, after accounting for the domestic load, is added to the utility grid is profiled as follows:

Denoting the total load demand by $P_{\text {Load }}$ and REG by $P_{P V}$ for a single household, the power is computed using (1):

$P(t)=V(t) \times I(t) \times \cos \varphi(t)$

where $V$ is the load or PV generated voltage, $I$ is the load or $\mathrm{PV}$ generated current and $\cos \varphi$ is the $\mathrm{PF}$ of the residential load. Moreover, the power difference from REG was calculated as:

$\Delta P(t)=P_{P V}(t)-P_{\text {Load }}(t)$

where if $\Delta P>0$, power would be supplemented to the utility and denoted by $\Delta P_{+}$and if $\Delta P<0$, the utility would provide the power deficit of the domestic load and denoted by $\Delta P_{-}$.

The calculated $\Delta P$, in watts, is converted into the standard unit (kWh) by:

$\operatorname{units}(t)=(\Delta P(t) \times T) / 1000$

with $T$ being the total time period the power is drawn into or from the utility. To compute the cost of energy differential, the local energy tariffs were used that are outlined below briefly. These computations, however, can be readily adapted for any tariff structure.

Although Pakistan has a centralized power distribution system spear headed by WAPDA (Water and Power Development Authority), regional power distribution companies adopt different tariff models. In accordance with local practices, the duty was calculated by a combination of block-rate and time-of-use (TOU) tariffs. Subsequent blocks of energy cost progressively higher depending on the units consumed, whereas, TOU tariff depends on the peak and off-peak hours of domestic load. However, the cost for domestic REG feeding to utility was according to a single-rate tariff [16]. Hence the two permutations for power differential were calculated as follows:

If $\Delta P>0$, with the fixed tariff of REG unit to utility as $x$ $P K R / k W h$, the total credit of REG owner $\left(O_{C}\right)$ for time period $\mathrm{T}$ is:

$O_{C}(t)=\frac{\Delta P_{+}(t) \times T}{1000} \times x$

Otherwise, with $\Delta P<0$, assuming a three-block model, for domestic load below its sanctioned load of $g \mathrm{~kW}$, fixed tariffs for respective blocks $a, b \& c$ were $P K R y, z \& v$ per $k W h$. For domestic load greater than the sanctioned load ( $g$ $k W)$, TOU tariffs were applied with different peak and offpeak rates. This study used $P K R$ w per $k W h$ peak and $P K R$ $p$ per $k W h$ off-peak TOU tariffs, with time periods of $h_{1} \&$ $h_{2}$ hours respectively. The total utility credit $\left(U_{C}\right)$ of energy consumed by REG owner above and below $g \mathrm{~kW}$ load demand for the time period $h_{1}$ and $h_{2}$ and $T=\left(T_{a}\right.$ 
$+T_{b}+T_{c}$ ) is expressed by equations $5 \& 6$, where $T>>$ $h_{1} \& h_{2}$ :

$$
\begin{aligned}
& U_{C, \text { Load }>g k W}(t)=\frac{g(t) \times h_{1}}{1000} \times w+\frac{g(t) \times h_{2}}{1000} \times p \\
& U_{C, \text { Load }<g k W}(t)=\frac{\left(\Delta P_{-}(t) \times T_{a}\right)}{1000} \times y+\frac{\Delta P_{-}(t) \times T_{b}}{1000} \times z+ \\
& \frac{\left(\Delta P_{-}(t) \times T_{C}\right)}{1000} \times v
\end{aligned}
$$

A concise flow of the complete net metering system is illustrated in figure 4. $C_{1}, C_{2} \& C_{3}$ are pre-defined control signals for net- metering control unit algorithm. Upon system initialization, the control signal $C_{1}$ indicates if the power available from the PV system is sufficient to fulfil the residential load. If the generated PV energy exceeds the load demand, the control signal $C_{2}$ is toggled high and subsequent energy calculations are performed using the uniform surplus energy model. Similarly, a control signal $C_{3}$ high is consistent with a surplus of the utility power available to fulfil the deficit PV energy of domestic load. Conversely, if the utility grid is unable to fulfil the power differential, the local battery storage system is programmed to provide the shortfall of PV energy. Finally, the cost of drawn and fed energy to and from the utility is calculated to surmise the aggregate monthly bill to the REG owner.

\section{B. Power Factor}

The REG and utility units for domestic loading only take into account real power tariff computations and assume the entire residential load to be a resistive load; hence, the PF calculations assist in calculating the accurate real power for R and RL domestic loadings and reveal the nature of domestic load (resistive / inductive). To determine the domestic PF, signal sampling was performed at $50 \mathrm{~Hz}$, with constant voltage and current signals from ZMPT101B ACS712 sensors, using 10-bit Node MCU $\mathrm{ADC}$ at $27 \mathrm{kHz}$ sampling frequency and resolution of 5 $\mathrm{V} / 1024 \mathrm{bit}$. Sampling values for resistive (R) and inductive (RL) loadings respectively are shown in figures $5 \& 6$ and were subsequently used to calculate the accurate RMS voltage, RMS current, real power (P), apparent power (S), and then the phase difference, as follows:

$I_{R M S}=\sqrt{\frac{\sum_{i=1}^{n} I_{i}^{2}}{n}}$

$V_{R M S}=\sqrt{\frac{\sum_{i=1}^{n} V_{i}^{2}}{n}}$

$P=\frac{1}{n} \sum_{i=1}^{n} V_{i} \times I_{i}$

$S=V_{R M S} \times I_{R M S}$

$\cos \varphi=\frac{P}{S}$

where $I_{i}$ and $V_{i}$ are the instantaneous sampled values of the current and voltage of the $i^{\text {th }}$ sampling, $I_{R M S}$ and $V_{R M S}$ are the RMS values of the current and voltage and $\mathrm{n}$ is the total number of samples. Tables $1 \& 2$ encapsulate some of the computed data for the aforementioned parameters.

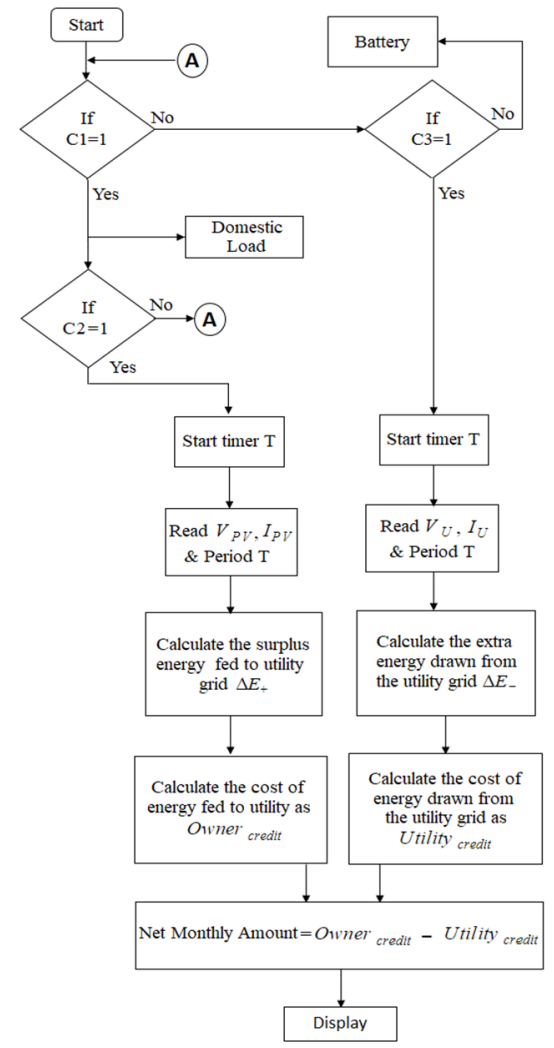

Fig. 4. Flow Graph for the Net Metering Operation

TABLE 1. PARAMETERS MEASURED BY THE NODE MCU (R-LOAD)

\begin{tabular}{|c|c|c|c|c|}
\hline$V_{R M S}$ & $I_{R M S}$ & $P$ & $S$ & $\cos \varphi$ \\
\hline 221.57 & 14.18 & 3140.91 & 3141.14 & 1.0 \\
\hline 220.91 & 14.25 & 3147.12 & 3147.97 & 1.0 \\
\hline 219.65 & 14.10 & 3096.85 & 3097.10 & 1.0 \\
\hline 221.02 & 13.93 & 3077.97 & 3078.26 & 1.0 \\
\hline
\end{tabular}

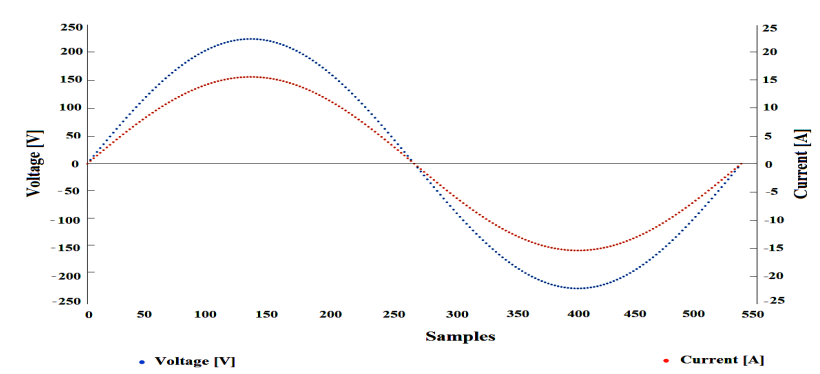

Fig. $5.50 \mathrm{~Hz}$ voltage and current signal sampling at $27 \mathrm{kHz}$ by the Node MCU demonstrating the stable nature of RMS power and 0 phase difference indicating resistive nature of load.

TABLE 2. PARAMETERS MEASURED BY THE NODE MCU (RL-LOAD)

\begin{tabular}{|c|c|c|c|c|}
\hline$V_{R M S}$ & $I_{R M S}$ & $P$ & $S$ & $\cos \varphi$ \\
\hline 220.06 & 4.23 & 734.21 & 931.82 & 0.79 \\
\hline 218.56 & 4.10 & 699.12 & 898.24 & 0.78 \\
\hline 221.35 & 3.96 & 702.7 & 876.28 & 0.80 \\
\hline 220.92 & 4.13 & 723.25 & 912.41 & 0.79 \\
\hline
\end{tabular}




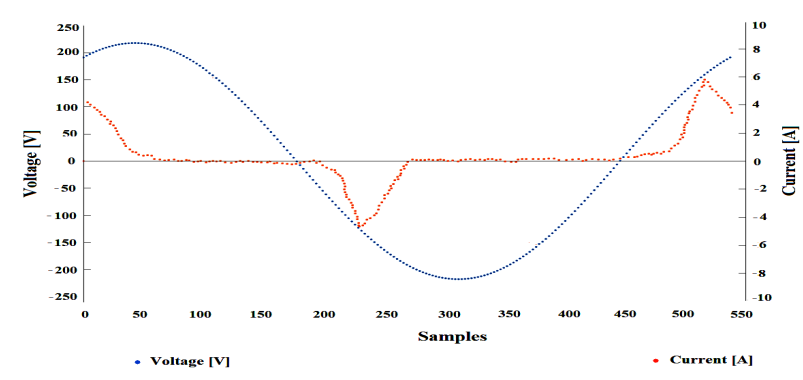

Fig. 6. $50 \mathrm{~Hz}$ voltage and current signal sampling at $27 \mathrm{kHz}$ by the Node MCU with the current lagging behind voltage showing RL nature of load and assisting with real power calculations.

\section{RESULT \& DISCUSSION}

To assess the performance of the proposed smart net metering system, a continuous 6-hour test, bifurcated equally in 3-hour periods, was conducted under variable loading, REG and utility tariff structures to simulate reallife operations on the Blynk IoT platform. Firstly, the surplus PV generation at domestic scale fed to the utility grid was measured and then, in the latter half, excess energy drawn from the utility under the presence of PV generation was monitored. All statistics were synchronously uploaded to the Blynk server wirelessly and readily made accessible for remote real-time control and analysis on the mobile app and as well as on the offline graphical control system, albeit independently but coincidentally.

\section{A. Results of PV to Utility Energy Export}

The 3-hour PV to utility energy profile is illustrated on Blynk IoT Super Cart, as shown in figure 7. The energy tariff for domestic REG units was calculated through a single-rate tariff model, with the feed-in-tariff set to 5 $(P K R) / k W h$. The maximum instantaneous power fed to the utility was $2.870 \mathrm{~kW}$ with an average power of $2.078 \mathrm{~kW}$. The net PV energy units fed to the utility, after accounting for power losses, totalled 125.89 corresponding to a positive credit of $615.3 P K R$. Since the gradient of unit and credit profiles depend solely on the instantaneous PV power shared to the utility, any fluctuations, for instance the considerable growth in energy units and credit occurring from 5:44 pm to $6: 00 \mathrm{pm}$, correspond to real-time variations in the PV generation. The simulated variable loading and intermittent PV generation led to sporadic instabilities in sensor measurements. Similarly, the smooth voltage profile of PV system demonstrates high-quality voltage regulation from the PV inverter, evident from figure 7 .

\section{B. Results of Utility to Consumer Energy Export}

The combined PV and utility energy profiles were studied for a domestic scale loading with the consumer energy tariffs expressed as combinations of block-rate and TOU tariffs. For domestic load below sanctioned limit, set to $2 \mathrm{~kW}$, the three blocks of $10 \mathrm{kWh}$ had tariffs of $5 \mathrm{PKR} /$ $k W h, 10 \mathrm{PKR} / \mathrm{kWh} \& 15 \mathrm{PKR} / \mathrm{kWh}$. However, if domestic load crosses the threshold of sanctioned limit, the peak and off-peak time tariffs were set to PKR 20/kWh \& PKR $12 / \mathrm{kWh}$ respectively, with a time interval of 2.5 minutes for each peak \& off-peak interval. The complete TOU and block-rate tariff structure is summarized in table 3 . The metering results are shown in figure 8 . With the initial gradient of the energy credit curve relatively flat, the point $R 1$ on figure 8 corresponds to the metering response at TOU tariff, which consequently results in a drastic increase in the slope of credit curve. Similarly, the points $R 2 \& R 3$ represent the switching of energy tariffs to the second \& third blocks respectively. The maximum instantaneous power demand from the utility was, over a duration of 60 seconds, $2.5 \mathrm{~kW}$ and the average power demand for the utility was $0.865 \mathrm{~kW}$. The total units consumed were 33.84 $k W h$ corresponding to a negative utility credit of 407.9 $(P K R)$. Thus, the aggregate credit amount for the 6-hour net metering operation was +207.4 (PKR), indicating a net profit for the domestic REG supplier

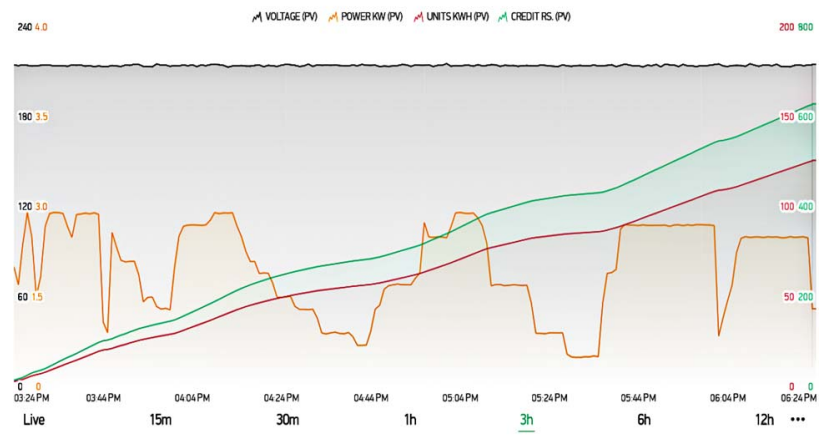

Fig. 7. Super Chart display for 3 hours PV generation

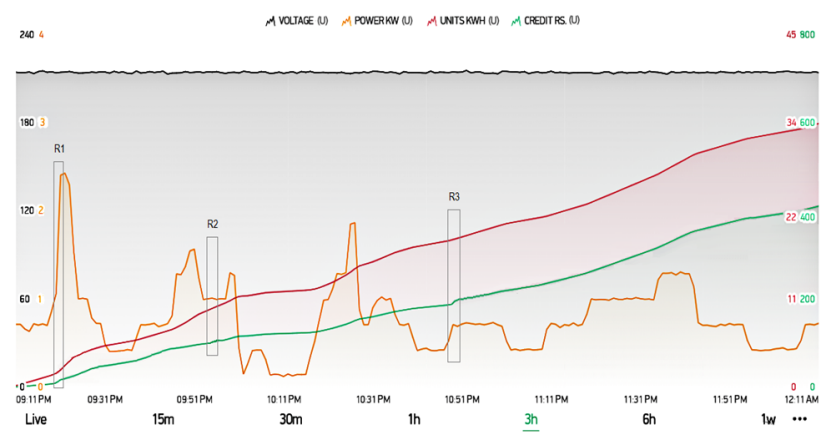

Fig. 8. Super Chart display for 3 hours combined PV and Utility generation

TABLE 3. TARIFF STRUCTURE FOR UTILTIY TO CONSUMER ENERGY TRADE

\begin{tabular}{|r|c|c|c|}
\hline $\begin{array}{r}\text { Sr } \\
\text { No. }\end{array}$ & Tariff Category & $\begin{array}{c}\text { Uniform Tariff Variable Charges } \\
\text { (PKR/KWh) }\end{array}$ \\
\hline a) & Sanctioned load $<2 \mathrm{~kW}$ & \multicolumn{2}{|c|}{} \\
\hline 1. & Load $<10 \mathrm{kWh}$ & \multicolumn{2}{|c|}{5.0} \\
\hline 2. & $10 \mathrm{~kW}<$ Load $<20 \mathrm{kWh}$ & \multicolumn{2}{|c|}{10.0} \\
\hline 3. & Load $>20 \mathrm{kWh}$ & \multicolumn{2}{|c|}{15} \\
\hline b) & Sanctioned load $>2 \mathrm{~kW}$ & \multicolumn{2}{|c}{} \\
\hline & & Peak & Off-Peak \\
\hline & Time of Use & 20.0 & 12.0 \\
\hline
\end{tabular}

\section{Wired \& Wireless Monitoring \& Control System}

The designed smart net metering system communicates with both circuited and wireless monitoring and control systems for better analysis and reliability. The online system consists of the IoT based Blynk mobile application which displays the real-time measured net metering computations in high-resolution graphic Blynk widgets and 
allows the PV owner to perform IoT based remote operations to terminate the supply of REG to the utility grid in case of technical faults or maintenance, whereas the offline system comprises of a high-definition LCD displaying data in sync with the Blynk App readings. (Figures 9 and 10).

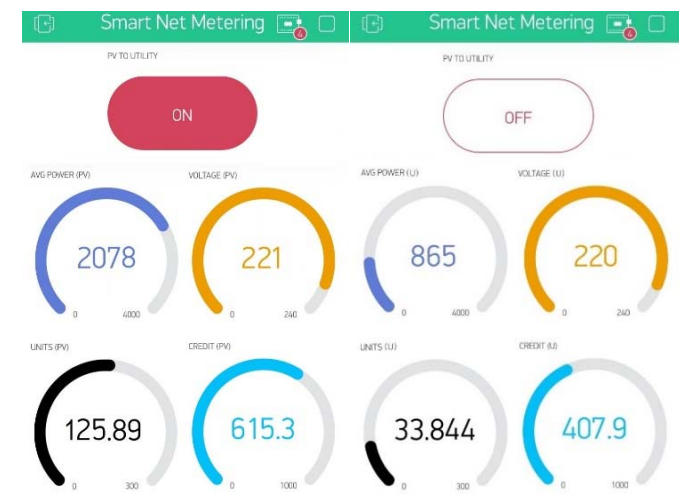

Fig. 9. Blynk mobile app widgets for PV and utility generation

The offline LCD displays the instantaneous value of voltage, PV to utility energy feeding status, cumulated energy units and the corresponding credits for PV to utility and utility to consumer energy trades. This offline display provides a reliable fail-safe in case of a potential connection loss to either the Blynk mobile app or the internet, a probable predicament considering the prevalent power shortfall in the sub-continent.

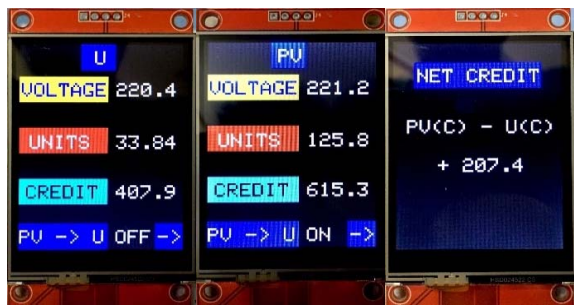

Fig. 10. Offline graphical touch LCD user display

\section{CONCLUSION}

The individual 3-hour net metering profiles of PV to utility and utility to consumer energy trade on the Blynk super chart showed precise and accurate credit readings under diverse tariff constraints and external simulations. Specifically, the momentous fluctuations in the PV generation and the tariff block shifts for the combined PV and utility power generation were sharply and distinctly expressed in the power chart, indicating swift detection capabilities of the net-metering system. Perhaps the integration of distinct visual/auditory signals to indicate these fluctuations can make the system more user-friendly in terms of signaling alerts. Finally, the offline and online databases were observed to sync, truthfully displaying both the instantaneous and aggregate calculations, without any considerable packet-loss or communication disruptions.

For future work we are considering the integration of industrial \& commercial tariff blocks into the algorithm for making the net-meter truly ubiquitous in every sense. However, owing to the complexity of these tariffs and the sheer amount of experimental data required along with the constraints for accuracy, we anticipate the process to take considerable testing time and resources. From there on, the next logical step is integration of an intelligent appliance control system with the smart IoT-based net-meter to offer a one-of-its-kind solution for decentralised power generation technologies.

\section{REFERENCES}

[1] Mishra, D. Irwin, P. Shenoy, J. Kurose and Ting Zhu, "Green Charge: Managing Renewable Energy in Smart Buildings", Journal on Selected Areas in Communications, vol. 31, no. 7, pp. 1281-1293, July 2013

[2] X. Fang, S. Misra, G. Xue and D. Yang, "Smart Grid - The New and Improved Power Grid: A Survey", Communications Surveys \& Tutorials IEEE, vol. 14, no. 4, pp. 944-980, 2012.

[3] A. Poullikkas, G. Kourtis, IoannisHadjipaschalis, "A review on net metering mechanism for electricity renewable energy resources", International journal of energy and environment, vol. 4, pp. 975-1002, 2013.

[4] S. Adnan, A. H. Khan, S. Haider, and R. Mahmood, "Solar energy potential in Pakistan" in Journal of Renewable and Sustainable Energy, 2012. DOI: 10.1063/1.4712051.

[5] Z. Al-Waisi and M. O. Agyeman, "Challenges and Opportunities of SmartMeters in Smart Homes and Smart Grids" in Proceedings of the 2nd International Symposium on Computer Science and Intelligent Control, pp. 1-6, 2018.

[6] Z. Jebroni, H. Chadli, B. Tidhaf, A. Benlghazi, and A. Tahani, "Gain correction and phase compensation of a smart electrical energy meter," in International Conference on Engineering MIS, pp. 1-6, 2016.

[7] M. C. Pacis , J. T. Sese, H. A. Blastique , M. D. C. Casibang, G. G. Ladisla and D. Villano, "Metering of Surplus Energy on PV Systems Using ZigBee Wireless Technology" in pro of IEEE International Conference on Control System, Computing and Engineering, 2016, pp. 348-353.

[8] Rahman, "Smart Net Energy Metering System" in Journal of Electrical \& Electronic Systems, 2018, DOI: 10.4172/23320796.1000285 .

[9] V. R. Deskar, V. Kumar, P. Kumar and S. Gururaj, "Design of Net Meter Using FPGA" in pro of IEEE International Conference on Recent Trends in Electronics Information Communication Technology, 2016, pp. 323- 326.

[10] K. Maharaja, P. P. Balaji, S. Sangeetha and M. Elakkiya, "Development of Bidirectional Net Meter in Grid Connected Solar PV System for Domestic Consumers" in pro of IEEE International Conference on Energy Efficient Technologies for Sustainability, 2016, pp. 46-49.

[11] C. F. Ostia, M. C. Ailes, V. P. G. Cantillon, B. L. Mangaoang, R. R. Sevilla and M. Pacis, "Development of a Smart Controller for Hybrid Net Metering" in pro of IEEE Region 10 Conference TENCON, 2017, pp. 1092-1096.

[12] W. Ali, H. Farooq, A. Khalid, A. Raza, and N. Tanveer, "Single phase GSM-based wire-less energy metering with a user notification system," in 2017 International Symposium on Wireless Systems and Networks, pp. 1-8, 2017.

[13] https://www.espressif.com/en/products/hardware/esp8266ex/overview

[14] https://blynk.io/en/developers

[15] V. K. Mehta and R. Mehta, Principles of Power System, 4th ed, pp. 87- 100, 1999.

[16] https://iesco.com.pk/index.php/customer-services/tariff-guide.

[17] H. Jamal, M. F. N. Khan, A. Anjum \& M. Janjua, "Thermal Monitoring and Protection for Distribution Transformer under Residential Loading using Internet of Things," IEEE Global Conference on Internet of Things, Dec, 2018.

[18] H. Jamal, M. Waseem, I. A. Sajjad, A. Anjum and M. S. Khan, "Low Cost Feedback Control Thermal Protection System for 3Phase Distribution Transformer using Microcontroller," in 6th IEEE International Conference on Smart Energy Grid Engineering, 2018, pp. 200-204.

[19] https://www.makerlab-electronics.com/product/ac-voltage sensormodule- 